\title{
Immunologic Susceptibility of Young Children to Mycobacterium tuberculosis
}

$\mathrm{A}^{\prime}$ $\mathrm{t}$ least one third of the world's population is infected with Mycobacterium tuberculosis (Mtb). Consequently, tuberculosis (TB) remains one of the most significant causes of childhood morbidity and mortality worldwide (1). Young children bear the burden of TB disproportionately. Once infected, children are not only more susceptible to TB than adults, but also are more likely to develop a severe form of the disease. Specifically, following infection more than $90 \%$ of immunocompetent adults will establish an asymptomatic, latent TB infection (LTBI), which carries a 5-10\% life-time risk of reactivation disease. Whereas, in the majority of young infants, a primary Mtb infection will advance to active TB, and then in a substantial proportion of those with active TB, the disease will progress to a more severe form (e.g., miliary TB). In addition to an increased susceptibility to TB, prompt diagnosis in children is complicated by the fact that children with progressive primary infections seldom present with a positive sputum acid-fast bacillus smear, which is commonly seen in adult pulmonary reactivation disease. Early detection is essential since progression of the disease occurs during the period of diagnostic delay. Understanding the basis for the increased susceptibility of children to developing TB is likely to lead to improved vaccines and immunodiagnostics.

Numerous studies in animal models and Mtb-infected adults have established innate and adaptive cellular immunity as playing an important role in preventing Mtb infection (2). The activated macrophage, which also is chief host cell for Mtb, serves as the first line of defense but is not sufficient for control of bacterial replication. Rather, containment depends on the priming of effective adaptive $\mathrm{T}$ cell responses by dendritic cells (DC). Studies using the murine TB model have established that $\mathrm{CD}^{+}{ }^{+} \mathrm{T}_{\mathrm{H}} 1$ cells and $\mathrm{CD}^{+} \mathrm{T}$ cells, which produce IFN- $\gamma$ and TNF- $\alpha$ and are cytolytic, are essential for host defense. In humans, healthy adults with LTBI demonstrate strong IFN- $\gamma$ producing $\mathrm{T}$ cell responses, whereas individuals immunocompromised by AIDS, or rare IFN- $\gamma$ receptor deficiencies, demonstrate increased susceptibility to TB. Furthermore, in adults, regulatory $\mathrm{T}$ cells have been shown to be associated with pulmonary $\mathrm{TB}$, and $\mathrm{CD}^{+} \mathrm{T}_{\mathrm{H}} 2$ cells, producing IL-4, IL-5, and/or IL-13, may be associated with more severe TB.

Immunologic immaturity is likely the cause for the high susceptibility of TB in children. Potential contributing factors to increased susceptibility include: 1) relatively impaired macrophage and DC function, 2) deficient quantity and/or delayed kinetics of effective $\mathrm{T}_{\mathrm{H}} 1$ cell and $\mathrm{CD}^{+} \mathrm{T}$ cell responses, 3) the propensity to develop $\mathrm{T}_{\mathrm{H}} 2$ over $\mathrm{T}_{\mathrm{H}} 1$ cell immunity, and/or 4) excessive $T$ regulatory cells (3). In this regard, early effective activation of innate immunity associated with the rapid and robust acquisition of $\mathrm{T}_{\mathrm{H}} 1$ immunity is likely to be of critical importance in children $(4,5)$. However, because there are few infant human studies of Mtb infection and no established infant TB animal models, much remains to be done to determine the specific aspect immunologic immaturity plays in TB susceptibility. A TB vaccine with improved efficacy in infants will require a better understanding of how infant and adult immunity differ following natural infection or bacille Calmette-Guérin vaccination. In regards to immunodiagnostics, the development of two $\mathrm{T}$ cell diagnostics for $\mathrm{Mtb}$ infection, the QuantiFERON assays and T-SPOT.TB, which measure T cell IFN- $\gamma$ release to Mtb antigens using an ELISA or ELISPOT format, respectively (6), represent a substantial advance in TB diagnostics, but will require more evaluation in children to improve diagnosis of childhood Mtb infection and TB. - Deborah A. Lewinsohn and David M. Lewinsohn

\section{REFERENCES}

1. Starke JR 2007 New concepts in childhood tuberculosis. Curr Opin Pediatr 19:306313

2. North RJ, Jung YJ 2004 Immunity to tuberculosis. Annu Rev Immunol 22:599-623

3. Lewinsohn DA, Gennaro ML, Scholvinck L, Lewinsohn DM 2004 Tuberculosis immunology in children: diagnostic and therapeutic challenges and opportunities. Int J Tuberc Lung Dis 8:658-674

4. Gold MC, Donnelly E, Cook MS, Leclair CM, Lewinsohn DA 2006 Purified neonatal plasmacytoid dendritic cells overcome intrinsic maturation defect with TLR agonist stimulation. Pediatr Res 60:34-37

5. Gold MC, Robinson TL, Cook MS, Byrd LK, Ehlinger HD, Lewinsohn DM, Lewinsohn DA 2007 Human neonatal dendritic cells are competent in MHC class I antigen processing and presentation. PLoS ONE 2:e957

6. Pai M, Dheda K, Cunningham J, Scano F, O'Brien R 2007 T-cell assays for the diagnosis of latent tuberculosis infection: moving the research agenda forward. Lancet Infect Dis 7:428-438 